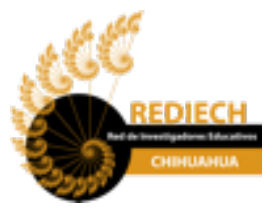

Red de Investigadores Educativos Chihuahua A.C. Chihuahua, México www.rediech.org

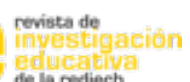

de la rediech

ISSN: 2007-4336

ISSN-e: $2448-8550$

http://www.rediech.org/ojs/2017/index.php/ie rie rediech/index

\author{
Ana María Orozco Santa María \\ Ma. Teresa García Ramírez \\ Luis Alberto Cepeda Villasana
}

2019

\title{
ALFABETIZACIÓN DIGITAL DESDE UN ENFOQUE INSTRUMENTAL, COGNITIVO Y EMOCIONAL EN ESTUDIANTES DE TURISMO USANDO BLACKBOARD
}

IE Revista de Investigación Educativa de la REDIECH, 10(19), pp. 11-35.

DOI: http://dx.doi.org/10.33010/ie_rie_rediech.v10i19.300

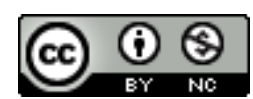

Esta obra está bajo licencia internacional Creative Commons Reconocimiento-NoComercial 4.0. 


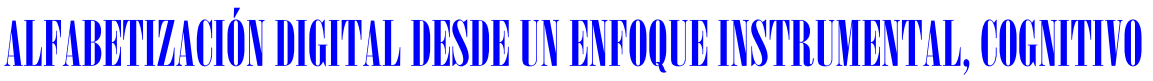

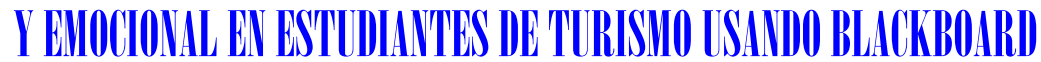

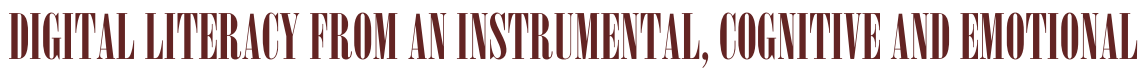

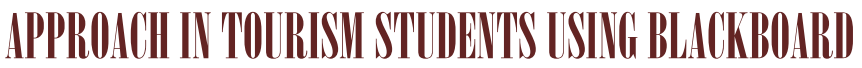

\author{
OROZCO SANTA MARÍA Ana María \\ GARCÍA RAMÍREZ Ma. Teresa \\ CEPEDA VILLASANA Luis Alberto
}

Recepción: septiembre 3 de 2018 | Aprobado para publicación: abril 29 de 2019

DOI: http://dx.doi.org/10.33010/ie_rie_rediech.v10i19.300

\section{Resumen}

Las universidades, conscientes de la importancia de formar ciudadanos competentes usando la tecnología para el estudio y el trabajo, han integrado asignaturas semipresenciales y totalmente en línea que favorecen la alfabetización digital. La investigación que se reporta analiza las

Ana María Orozco Santa María. Profesora de las Escuelas de Turismo y Gastronomía de la Universidad Anáhuac, Querétaro, México. Es maestra en Educación y cursa el Doctorado en Tecnología Educativa en la Universidad Autónoma de Querétaro. Es miembro del comité académico del EGEL-TUR del Ceneval. Entre sus publicaciones recientes se encuentran Autopercepción de habilidades de aprendizaje en ambientes virtuales (2017) y Foros de discusión: herramienta que promueve la presencia cognitiva, social y de enseñanza (2018). Trabaja las líneas de investigación alfabetización digital y foros de discusión. Correo electrónico: anaor8555@gmail.com. ID: https://orcid.org/00000003-3358-827X.

Ma. Teresa García Ramírez. Profesora-investigadora de la Facultad de Informática de la Universidad Autónoma de Querétaro, México. Es doctora en Tecnología Avanzada por el Instituto Politécnico Nacional y tiene reconocimiento al perfil Prodep. Entre sus publicaciones recientes en coautoría con sus tesistas se encuentra Percepción del uso de TIC en matemáticas bajo el enfoque de competencias en educación superior (2017) y coordinadora del libro Escenarios y desafíos de la tecnología educativa (2016). Ha dirigido tesis de licenciatura y posgrado. Trabaja la línea de investigación impacto de las TIC en el desarrollo de modelos y entornos educativos. Correo electrónico: teregar@uaq.mx. ID: https://orcid.org/0000-0002-5524-2002.

Luis Alberto Cepeda Villasana. Profesor de tiempo completo en la Escuela de Finanzas y Contaduría Pública de la Universidad Anáhuac, Querétaro, México. Es maestro en economía con especialidad en economía industrial. Ha trabajado en distintos cargos del sector público y privado aplicando los métodos estadísticos para la solución de problemas aplicados en el área fiscal, poblacional y en investigaciones de mercado. Entre sus publicaciones recientes se encuentran Gasto familiar y remesas. Un instrumento fracasado para atenuar la pobreza (2017). Trabaja las líneas de investigación desarrollo económico, economía de la educación y análisis financiero. Correo electrónico: luis. cepeda@anahuac.mx. ID: https://orcid.org/0000-0002-9946-0503. 
dimensiones instrumental, cognitiva y emocional de la alfabetización digital de estudiantes de turismo que cursan materias en línea en Blackboard. El diseño de este estudio fue no experimental transeccional para explorar la percepción de los estudiantes sobre su aprendizaje usando Blackboard, y correlacional para obtener una medida de la relación entre las dimensiones. El principal hallazgo mostró que los alumnos perciben que aprenden mejor en cursos presenciales que los correspondientes en línea. Esto podría indicar que los estudiantes no han aceptado ni adoptado a Blackboard como un medio para su aprendizaje.

\title{
Palabras clave: ALFABETIZACIÓN DIGITAL, DIMENSIÓN INSTRUMENTAL, DIMENSIÓN COGNITIVA, DIMENSIÓN EMOCIONAL, BLACKBOARD.
}

\begin{abstract}
Universities, aware of the importance of training competent citizens in the use of technology for study and work, have integrated blended and fully online subjects that favor digital literacy. The research reported analyzes the instrumental, cognitive and emotional dimensions of digital literacy of tourism students using Blackboard. The design of this study was based on a non-experimental transactional model to explore students' perception of their learning using Blackboard, and correlational to obtain a measure of the relationship between dimensions. The main finding showed that students perceive that they learn better in face-to-face courses than in online courses. This could indicate that students have not accepted or adopted Blackboard as a means of learning.
\end{abstract}

Keywords: DIGITAL LITERACY, INSTRUMENTAL DIMENSION, COGNITIVE DIMENSION, EMOTIONAL DIMENSION, BLACKBOARD.

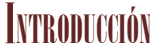

\section{Ser individuos digitalmente alfabetizados es una prioridad en un} entorno en donde se requiere usar una amplia variedad de dispositivos electrónicos, aplicaciones y programas. El uso de la tecnología en los procesos de enseñanza-aprendizaje en educación superior implica un compromiso social con la calidad de la educación tanto para docentes como para estudiantes. Conscientes de esta necesidad, países como Chile, Colombia, Costa Rica, México, Perú, Uruguay y Venezuela han diseñado programas y proyectos internacionales para desarrollar habilidades para usar la tecnología (IIPE-UNESCO, 2006). La integración de las tecnologías de la información y la comunicación (TIC) en las universidades como complemento a la educación presencial, en programas semipresenciales y en línea es ya una realidad.

Los cursos en línea que ofrecen universidades y centros especializados en México 12 han aumentado. La Asociación Nacional de Universidades e Instituciones de Educa- 
ción Superior (ANUIES) reportó 66 programas de educación formal en el año 2001 y 407 programas en el 2015. Ante el crecimiento de la oferta educativa en modalidad a distancia, que se hace evidente al contrastar ambas fuentes de información, surge la necesidad de formar a los estudiantes para desenvolverse en un ambiente virtual para el que no han recibido una capacitación formal.

Los docentes del siglo XXI forman estudiantes que son nativos digitales y que poseen características distintas a otras generaciones. Los procesos educativos se han permeado de suposiciones sobre lo que saben hacer en el ámbito tecnológico y con esos supuestos como guía se ha pensado que no tienen necesidad de una formación digital.

Es bien conocido que los nativos digitales disfrutan usando diferentes herramientas tecnológicas; sin embargo, es importante resaltar que usar la tecnología para el entretenimiento es diferente que para el aprendizaje. Benavides y Pedró (2007) señalan que la investigación educativa tiene que cubrir la necesidad de constatar el impacto que tienen las TIC en los alumnos. Con esta idea, el presente estudio plantea la importancia de analizar las dimensiones instrumental, cognitiva y emocional de la alfabetización digital de estudiantes de turismo de una universidad privada que cursan materias usando Blackboard para poder diseñar estrategias que favorezcan sus habilidades para usar un entorno virtual de enseñanza-aprendizaje (EVEA).

\section{ArTecheneiths}

Jimoyiannis y Gravani (2011) afirman que las TIC desempeñan un papel central y dominante en nuestras vidas y tienen gran influencia en diferentes aspectos tales como la educación, el trabajo, los servicios públicos, el entretenimiento y la cultura. En la misma tesitura, Lynch (2017) considera que la importancia de la tecnología en la sociedad convierte a la alfabetización digital en la herramienta de aprendizaje más importante para toda la vida. Por tal razón, la integración de la tecnología en diferentes niveles y modalidades de enseñanza es fundamental.

Diferentes organizaciones e investigadores han estudiado los avances en el uso de las TIC para actividades educativas. Alfabetizar digitalmente es una responsabilidad $\mathrm{y}$ un reto para las universidades en México, y todas las personas encargadas de planificar, diseñar e implementar programas en línea deberían considerar que "los medios por sí solos no provocan cambios significativos ni en la educación en general, ni en los procesos de enseñanza-aprendizaje en particular" (Cabero y Barroso, 2015, p. 49).

La International Association for the Evaluation of Educational Achievement, a través del International Computer and Information Literacy Study (ICIL), realizó la evaluación de las habilidades de 60,000 estudiantes de octavo grado en más de 3,300 escuelas de 21 sistemas educativos. Por parte de Latinoamérica participaron la Ciudad de Buenos Aires (Argentina) y Chile. El estudio demostró que la suposición de que los estudiantes están familiarizados con las TIC no es correcta. El 83\% alcanzó al menos el nivel 1, que indica un conocimiento mínimo de software básico, y solamente $2 \%$ logro el nivel 4 , que requiere aplicar el pensamiento crítico en la búsqueda de información (IEA, 2014). 
Otras investigaciones con estudiantes latinoamericanos muestran que: a) existe diferencia entre el conocimiento autopercibido sobre competencias digitales y los resultados de preguntas prácticas (ICDL Américas, 2015); b) no se apropian del potencial de la tecnología más allá de los usos básicos o recreativos (Berrío Zapata y Rojas, 2014); c) presentan deficiencia en el uso de la tecnología para los estudios (Godoy-Rodríguez, 2009); y, d) muchas actividades digitales realizadas dentro de un contexto informal no resultan ser saberes tan expertos, o bien no son trasladables a otros contextos (Bossolasco y Storni, 2012).

Van den Beemt (2010) señala que la investigación empírica cuestiona seriamente la premisa de una generación hábil y homogénea, ya que, en algunos estudios, se ha hecho evidente que los jóvenes tienen un nivel intermedio de habilidades en el uso de las TIC y que al usar internet muestran formas mundanas de comunicación y de recuperación de información en lugar de mostrar innovación y creatividad.

Las investigaciones aportan información valiosa que avala la sugerencia de Koutropoulos (2011) sobre el riesgo de seguir utilizando los nombres "generación digital", "net" o "Google" debido a que esos términos no los describen y pueden evitar que logren un crecimiento personal al asumir que ya poseen ciertas habilidades que en realidad no tienen.

\section{Entornos virtuiles de enseñintiit-ipprendizitije}

El espacio en el que pueden encontrarse profesores y estudiantes en la educación a distancia ha recibido diferentes nombres, entre los que se encuentran Learning Management System (LMS), Learning Content Management System (LCMS) y Virtual Learning Environment (VLE). En español se conocen como sistema de gestión de aprendizaje (SGA), entorno virtual de aprendizaje (EVA), entorno virtual de enseñanza-aprendizaje (EVEA) y ambiente virtual de aprendizaje (AVA). Para propósitos de esta investigación, se utilizará el término "entorno virtual de enseñanza-aprendizaje".

Barker y Gossman (2013) mencionan que es difícil precisar desde cuándo se usan los EVEA, pero que desde los años setenta, la Universidad Abierta del Reino Unido utilizó computadoras para el aprendizaje. En México, universidades tanto públicas como privadas han implementado programas diseñados para EVEA desde la década de 1990.

Hiraldo (2013) define el EVEA como "el conjunto de medios de interacción sincrónica y asincrónica, donde se lleva a cabo el proceso enseñanza y aprendizaje, a través de un sistema de administración de aprendizaje" (p. 1). Asimismo, señala que constituye un escenario para un aprendizaje autodirigido con el apoyo de recursos para un aprendizaje activo que permite facilitar la construcción de conocimientos y la adquisición de competencias.

Un EVEA se "emplea para crear, aprobar, administrar, almacenar, distribuir y gestionar las actividades de formación virtual" (Clarenc et al., 2013, p. 29). Resaltan que el conjunto de funciones de un EVEA no solo debe crear el espacio para el aprendizaje, sino también las condiciones para que el aprendizaje sea una verdadera experiencia. 
En México, un EVEA utilizado principalmente en instituciones de educación privadas es la plataforma comercial conocida con el nombre de Blackboard, empresa fundada en 1997 por dos asesores educativos, Matthew Pittinsky y Michael Chasen. Ofrece como ventajas la adaptación del producto a las necesidades del cliente, un soporte profesional y cuatro áreas primarias para los docentes: administración de contenidos, comunicación, evaluación y control, que permiten desarrollar habilidades digitales.

\section{Aliabetizición digital}

Gilster (1997) introdujo el término alfabetización digital en su libro del mismo nombre y no proporcionó un listado de competencias que establecieran lo que significa ser digitalmente alfabetizado. Explicó que se trataba de la habilidad para comprender y usar información de diversas fuentes digitales.

La Organización para la Cooperación y el Desarrollo Económicos (OCDE) destaca que la alfabetización digital "no se limita a realizar tareas sencillas con el ordenador" (OCDE, 2002, p. 100), y aclara que es necesario comprender el potencial de la tecnología y tener la habilidad y la confianza para adoptar las aplicaciones convenientes. Asimismo, propuso una tipología para las habilidades relacionadas con las TIC que incluye habilidades funcionales para un uso adecuado de las diferentes aplicaciones, habilidades para aprender (producto de la combinación de lo cognitivo con lo funcional) y habilidades propias del siglo XXI indispensables en la sociedad del conocimiento (OCDE, 2010).

La Organización de las Naciones Unidas para la Educación, la Ciencia y la Cultura (UNESCO, 2013, p. 16) resalta que es fundamental comprender que las TIC "no son solo herramientas simples, sino que constituyen sobre todo nuevas conversaciones, estéticas, narrativas, vínculos relacionales, modalidades de construir identidades y perspectivas sobre el mundo". Para no ser una persona socialmente excluida se deben poseer habilidades básicas relacionadas con las TIC que podrán obtenerse con proyectos de formación en alfabetización digital.

Otra definición internacionalmente conocida es la propuesta por una compañía sin fines de lucro del Reino Unido llamada Joint Information Systems Committee, JISC (2014), que explica que las alfabetizaciones digitales se integran por las capacidades que permiten a un individuo vivir, aprender y trabajar en una sociedad digital.

Para alfabetizar digitalmente existen diferentes modelos (Area, 2015; Belshaw, 2011; Gutiérrez, 2003; Hoobs, 2010; JISC, 2014; Payton y Hague, 2010) que especifican las habilidades que un individuo debe poseer para aprovechar de manera eficiente las TIC en diferentes actividades. En el presente estudio se exploraron las dimensiones instrumental, cognitiva y emocional por las repercusiones que generan en el rendimiento académico de los estudiantes que cursan materias en línea usando Blackboard. Además, se indagó sobre las dificultades con las herramientas este mismo sistema y la preferencia sobre otras herramientas tecnológicas. 


\section{llimensión instr'unential}

Los avances de la tecnología implican que los ciudadanos del siglo XXI adquieran los conocimientos y habilidades necesarios para trabajar con aplicaciones informáticas básicas usando una computadora personal. Es así como el manejo de lo instrumental constituye una de las dimensiones de la alfabetización digital. Area (2012, p. 30) la define como la "relativa al dominio técnico de cada tecnología y de sus procedimientos lógicos de uso".

Poblete y Villa (2013, p. 167) refieren que, al revisar las competencias de más de veinte instituciones educativas, una de las trece más frecuentes es el uso de las TIC que "se relaciona con la gestión de la información y de la comunicación apoyada en las amplias tecnologías a las que da acceso el ordenador personal". Las computadoras se han convertido en un artículo esencial para el trabajo y para el estudio, por lo que adquirir "el conjunto de conocimientos, habilidades, disposiciones y conductas que capacitan a los individuos para saber cómo funcionan las TIC, para qué sirven y cómo se pueden utilizar para conseguir objetivos específicos" (CRUE-TIC y REBIUN, 2009, p. 7) es indispensable para desenvolverse en el mundo digital.

Para usar correctamente el hardware y el software es necesario adquirir lo que se conoce como competencias informáticas. Prieto, Lloris y Torres $(2002$, p. 1) mencionan que la informática "trata de la adquisición, representación, almacenamiento, tratamiento y transmisión de la información". Las computadoras permiten capturar, procesar y presentar información a través de programas que han sido diseñados para seguir el razonamiento humano.

Blackboard cuenta con diferentes herramientas que los estudiantes deben dominar técnicamente para lograr una experiencia satisfactoria en el proceso de aprendizaje. Dichas herramientas son las siguientes: 1) anuncios, que permiten al docente comunicar información importante sobre diferentes tópicos; 2) calendario, cuya función es registrar los eventos del curso; 3) mis calificaciones, en donde se muestran los resultados obtenidos por el estudiante y la retroalimentación generada por el docente sobre las actividades de aprendizaje; 4) correo electrónico, que permite la comunicación con todos los estudiantes inscritos en un curso; $y, 5$ ) información personal, para crear una imagen académica a través de un perfil que puede editarse y compartirse con el grupo. Asimismo, los estudiantes pueden participar en conferencias web a través de Blackboard Collaborate, trabajar en equipo al formar parte de un grupo creado por el docente y participar en los tableros de discusión.

\section{Dimensión coggnitivi}

Area (2012, p. 30) explica que la dimensión cognitiva implica "aprender a utilizar de forma inteligente la información para acceder a la misma, otorgarle significado, analizarla críticamente y reconstruirla personalmente". También incluye la comunicación usando recursos digitales.

La cognición se refiere a la facultad que poseen los seres humanos para procesar información. Rivas (2008, p. 77) explica que la cognición "entraña procesos 16 de adquisición, transformación, organización, retención y uso de la información". 
Lo anterior implica que los estudiantes universitarios deben extraer información de Blackboard de manera activa, procesarla y usarla para adquirir nuevos conocimientos.

El sistema cognitivo humano incluye procesos que actúan en diferentes niveles. En el nivel bajo se ubican los procesos sensoriales, perceptivos y atencionales. En un nivel intermedio están el aprendizaje, la memoria y la comunicación oral y escrita. Y en el nivel alto el razonamiento, la creatividad, la toma de decisiones y la solución de problemas (Sanz de Acedo, 2010). La cognición permite integrar la información que se adquiere a través de los sentidos para crear estructuras del conocimiento, por lo que los estudiantes deben desarrollar habilidades para la adquisición, organización, retención y uso del conocimiento.

El origen y la definición de cognición aporta datos que permiten comprender la relevancia de la dimensión cognitiva en la alfabetización digital. Gilster (1997) mencionó la habilidad para comprender información; ser capaces de evaluarla e interpretarla es crucial. Bawden (2008) retomó esta afirmación y estableció cuatro componentes para la alfabetización digital: 1) fundamentos, los relativos a la alfabetización tradicional y a la alfabetización de la tecnología de información y comunicación; 2) conocimientos previos, aquellos que forman parte de una persona educada y que se obtienen a través de medios impresos; 3) competencias centrales, entre las que se encuentran leer y entender formatos digitales y no digitales, crear y comunicar información digital, evaluar información, construcción del conocimiento, alfabetización informacional y alfabetización mediática; y, 4) actitudes y perspectivas, de gran importancia para vincular el concepto de alfabetización digital con la idea más antigua de alfabetización (simplemente leer y escribir).

\section{Dimensión emocionial}

Las emociones son indicadores de la forma en que los individuos y grupos asimilan los contenidos culturales al mostrar ya sea compromiso o rechazo. Deben comprenderse como "portadoras de interpretaciones y significados dependientes de consideraciones sociales y culturales que definen los momentos y las circunstancias en que debe ser experimentada cada una de ellas y con qué grado de intensidad debe hacerse" (Rodríguez, 2008, p. 148).

En el campo educativo, Mora (2013) ha señalado que las emociones son muy importantes para dar sustento a los procesos de aprendizaje y sirven para almacenar y evocar memorias de forma efectiva. Ante tal afirmación, las emociones que se generan al usar las TIC intervienen en la actitud que tendrán los estudiantes al enfrentarse a nuevas herramientas. Dichas emociones se evidencian en comportamientos positivos o negativos que pueden favorecer o desalentar la apropiación tecnológica para uso crítico y productivo.

Como lo señalan Thüring y Mahlke (2007, p. 254), en la interacción humano-tecnología ya no solo son importantes la utilidad y la usabilidad, sino también factores como las cualidades estéticas y la experiencia emocional. Los resultados de la investigación que llevaron a cabo demostraron que "la manipulación de unas propiedades selectivas del sistema puede conducir a diferencias de usabilidad, lo cual afecta las reacciones emocionales del usuario". En sus experimentos usaron 
dos versiones de dispositivos tecnológicos para estudiar las reacciones de los participantes en el estudio. Confirmaron que la experiencia del usuario se compone de emociones-percepciones y de cualidades instrumentales-no instrumentales.

Tanto el aprendizaje como las experiencias en entornos digitales provocan emociones que influyen en los procesos educativos. Debido a ello, Area (2015, p. 5) incluye el componente emocional como una de las dimensiones implicadas en la alfabetización digital y la define como "la relativa al conjunto de afectos y emociones provocadas por la experiencia en entornos digitales". Esta dimensión propone un equilibrio afectivo-personal en el uso de las TIC e involucra el control de las emociones negativas, el desarrollo de la empatía y la construcción de una identidad digital.

Moore y Kuol (2007) reportaron que estudiantes universitarios asociaban estados emocionales positivos con buena enseñanza y buen aprendizaje, además de que se referían a emociones y experiencias emocionalmente satisfactorias al describir una enseñanza excelente. Estudios longitudinales en nueve países pertenecientes a la OCDE han mostrado que, además de las habilidades cognitivas y sociales, las emocionales son significativas para mejorar los resultados económicos y sociales (OCDE, 2015).

Wosnitza y Volet (2005) señalan que, en un ambiente en línea, las emociones están dirigidas a la persona, a la tarea 0 a la tecnología. Por ejemplo, el gozo experimentado al progresar en el aprendizaje o la frustración por no poder solucionar problemas se asocian con la persona; la alegría o el enojo derivados de la facilidad 0 dificultad para realizar una tarea se vinculan con la tarea; y las emociones dirigidas a la tecnología se relacionan con la capacidad del equipo de cómputo utilizado.

En síntesis, las emociones son muy importantes para dar sustento a los procesos de aprendizaje y sirven para almacenar y evocar memorias de forma efectiva (Mora, 2013). El ambiente emocional conformado, entre otros factores, por lo que el maestro dice y hace, los materiales y cómo se usan y las actividades que los estudiantes realizan contribuyen al estado de aprendizaje de los estudiantes (Mortiboys, 2016).

\section{Nlerenoulocil}

El diseño del presente estudio fue no experimental transeccional exploratorio para conocer la percepción de los estudiantes sobre su aprendizaje usando Blackboard. Además, para obtener una medida de la relación entre las dimensiones instrumental, cognitiva y emocional se realizó un análisis correlacional.

Se seleccionó a estudiantes de turismo debido a que las TIC tienen un valor estratégico en la formación de profesionales para las diferentes empresas del sector. La economía turística es impulsada por la tecnología, y empresas como las agencias de viajes, los touroperadores, renta de autos, cruceros y hoteles experimentan el creciente impacto que tienen en su operación (Elżbieta, 2016).

Se indagó sobre la existencia de cuestionarios sobre las habilidades relativas a las dimensiones de la alfabetización digital usando Blackboard en la universidad. Dado que no se encontró ningún instrumento previo que contribuyera al objetivo 18 planteado, se elaboró uno propio, de acuerdo con lo propuesto por Bernal (2016). Se 
determinó que el tipo de preguntas idóneas serían las de respuesta a escala sobre la percepción del aprendizaje vinculado con las dimensiones instrumental, cognitiva y emocional de la alfabetización digital.

Se realizó una prueba piloto para validar el instrumento donde participaron 30 estudiantes de carreras de ciencias sociales. Se obtuvo un alfa de Cronbach de 0.89, con lo cual se asegura que el instrumento es fiable. Asimismo, se solicitó el apoyo de tres profesoras con una experiencia mayor a cinco años en educación en línea y diseño instruccional para llevar a cabo un juicio de expertos. Sus opiniones y comentarios se recabaron en una hoja de registro y se realizaron los ajustes necesarios a las preguntas del cuestionario.

El instrumento para realizar el diagnóstico de las dimensiones instrumental, cognitiva y emocional de la alfabetización digital se compone de seis secciones:

1. Información demográfica. En esta sección se incluyen preguntas sobre el semestre cursado, institución en donde estudiaron la preparatoria, sexo, edad y cantidad de cursos semipresenciales y en línea que han tomado.

2. Dimensión instrumental. Consta de 15 preguntas para explorar la autopercepción sobre las habilidades relativas al dominio técnico de Blackboard.

3. Dimensión cognitiva. Incluye 15 preguntas sobre procesos relacionados con la adquisición, transformación, organización y uso de la información.

4. Dimensión emocional. Contiene 14 preguntas sobre las emociones experimentadas al usar Blackboard como plataforma de aprendizaje.

5. Herramientas tecnológicas. Consta de cinco preguntas sobre sus preferencias para usar libros electrónicos, videos, tutoriales, páginas web y podcast.

6. Aprendiendo en línea. Incluye 13 preguntas sobre las dificultades que enfrentan al usar herramientas de Blackboard y sobre otras disponibles en la red para realizar trabajos colaborativos en línea, guardar archivos en la nube, comunicarse con sus maestros y compartir imágenes en un tablero digital.

Para las secciones 2, 3, 4 y 5 se usó una escala de Likert con cinco niveles, desde totalmente en desacuerdo hasta totalmente de acuerdo. En la sección 6 se usó una escala de Likert con cinco niveles, desde nunca a muy frecuentemente.

El estudio fue realizado con los resultados del cuestionario aplicado a alumnos de la carrera de turismo de una universidad privada del centro de la República Mexicana que han usado Blackboard como EVEA en al menos una materia. Participaron 53 estudiantes, de los cuales 42 eran mujeres y 11 hombres. La edad promedio fue de 20 años. El estudio se realizó de enero a diciembre de 2017.

\section{Resilitinos}

Los objetivos de este estudio fueron explorar la percepción sobre las dimensiones instrumental, cognitiva y emocional que tienen los estudiantes de turismo matriculados en cursos mediados por Blackboard y obtener una medida de la relación entre las dimensiones. 


\section{limensión instr'tumential}

Ricoy y Fernández (2013, p. 521) refieren que "el principal inconveniente que encuentran los participantes para utilizar las TIC es de tipo técnico", por lo que, tomando en cuenta dicha dificultad, se exploró la percepción sobre el dominio de dos herramientas para la información y la comunicación disponibles en Blackboard. Se indagó si los estudiantes usan y conocen cómo usar el correo electrónico y cómo registrar información personal dentro de su perfil para presentarse ante sus compañeros y profesores.

En lo relativo al correo electrónico, $6 \%$ aceptó que no sabe usarlo y solamente el $45 \%$ lo utiliza para comunicarse con sus maestros (ver figura 1). Tales datos merecen atención, considerando que la mensajería electrónica no es algo novedoso o desconocido por los jóvenes, ya que usualmente se comunican con cuentas de proveedores como Hotmail, Gmail y Yahoo.

El uso del correo electrónico en la educación es versátil y tiene tres funciones: informativa, didáctica y comunicativa (Puerta Gil y Sánchez Upegui, 2010). Ofrece diferentes beneficios a la educación, entre los que se encuentran: desarrollo de habilidades comunicativas, crear conciencia sobre la acción de redactar textos claros, estimular la cultura de colaboración, desarrollar la autonomía en los procesos de aprendizaje y la accesibilidad inmediata a información de interés (Pérez, 2009).

Blackboard también ofrece herramientas sociales que facilitan crear una identidad y buscar a otros usuarios. Para crear una identidad, los estudiantes pueden ingresar datos dentro del perfil para presentarse ante sus compañeros de clase y profesores. En el perfil se puede incluir una fotografía y datos como los estudios que realiza, intereses académicos y profesionales, preferencias relativas a actividades recreativas y dirección de correo electrónico. El perfil se puede conectar con redes sociales como Facebook y Twitter y permite establecer opciones de privacidad. Los perfiles creados se almacenan en la nube de Blackboard y pueden conservarse durante toda la vida académica y profesional del usuario.

En educación a distancia, la publicación de información dentro del perfil es básica para la comunicación y la interacción entre los diferentes usuarios de un curso. Dado que no hay sesiones presenciales, la forma de conocer a las personas es a través de lo que se publica en el perfil.

Fig. 1. Utilizo el correo electrónico de Blackboard para comunicarme con mis maestros.

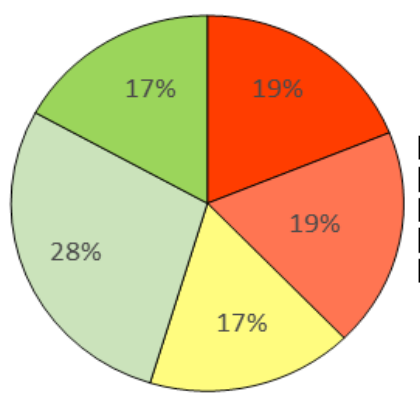

Totalmente en desacuerdo

En desacuerdo

$\square$ Ni de acuerdo ni en desacuerdo

De acuerdo

Totalmente de acuerdo

Fuente: Elaboración propia. 
Salman (2015) explica que la naturaleza del aprendizaje en línea provee una estructura para interacciones digitales y un ambiente seguro para que los estudiantes puedan desarrollar o mejorar sus identidades digitales relacionadas con actividades educativas. La identidad digital puede definirse como la suma de toda la información digital disponible acerca de un individuo (Boston Consulting Group, 2012). Lo que alguien publica sobre sí mismo dejará una huella digital y las universidades, a través de la creación de sus perfiles en los EVEA, podrían concientizar a los alumnos sobre lo que significa ser ciudadanos digitales.

El estudio mostró que solamente el $8 \%$ reconocieron haber editado su perfil en Blackboard, lo que podría indicar tres situaciones: no conocen cómo ingresar la información, tienen temor de publicar información por cuestiones de seguridad o no conocen la importancia de ir construyendo una identidad digital sólida y profesional desde su formación universitaria (ver figura 2).

Para determinar los efectos que cada dimensión estudiada tiene sobre las demás, se realizó un análisis correlacional en donde también se incluyeron las dos secciones sobre herramientas tecnológicas educativas y aprendiendo en línea. El análisis de la dimensión instrumental se muestra en la tabla 1.

Interpretación de las correlaciones de la dimensión instrumental (para tener un panorama completo en cada una de las dimensiones, es importante resaltar que las interpretaciones se repetirán en las secciones correspondientes):

1. DI-DC (0.518). Se observó que ambas dimensiones tienen un grado de correlación moderado, que podría indicar que el dominio técnico de una tecnología (en este caso Blackboard) facilitará el uso inteligente de la información. Dicha correlación es positiva, lo que implica también que ambas dimensiones se fortalecen mutuamente. A medida que los alumnos se consideran buenos con el uso de la

\begin{tabular}{lccccc}
\hline & \multicolumn{4}{l}{ Tabla 1. Resultados de las correlaciones de la dimensión instrumental } \\
\hline & & $\begin{array}{c}\text { Dimensión } \\
\text { cognitiva }\end{array}$ & $\begin{array}{c}\text { Dimensión } \\
\text { emocional }\end{array}$ & $\begin{array}{c}\text { Herramientas } \\
\text { tecnológicas }\end{array}$ & $\begin{array}{c}\text { Aprendiendo } \\
\text { en línea }\end{array}$ \\
\hline \multirow{2}{*}{$\begin{array}{l}\text { Dimensión } \\
\text { instrumental }\end{array}$} & $\begin{array}{c}\text { Correlación de } \\
\text { Pearson }\end{array}$ & $0.518^{* *}$ & $0.527^{* *}$ & $0.321^{*}$ & 0.260 \\
\cline { 2 - 6 } & Sig. (bilateral) & 0.000 & 0.000 & 0.019 & 0.060 \\
\cline { 2 - 6 } & $\mathrm{N}$ & 53 & 53 & 53 & 53 \\
\hline
\end{tabular}

*Significativa al 0.05 bilateral. **Significativa al 0.01 bilateral.

Fuente: Elaboración propia.

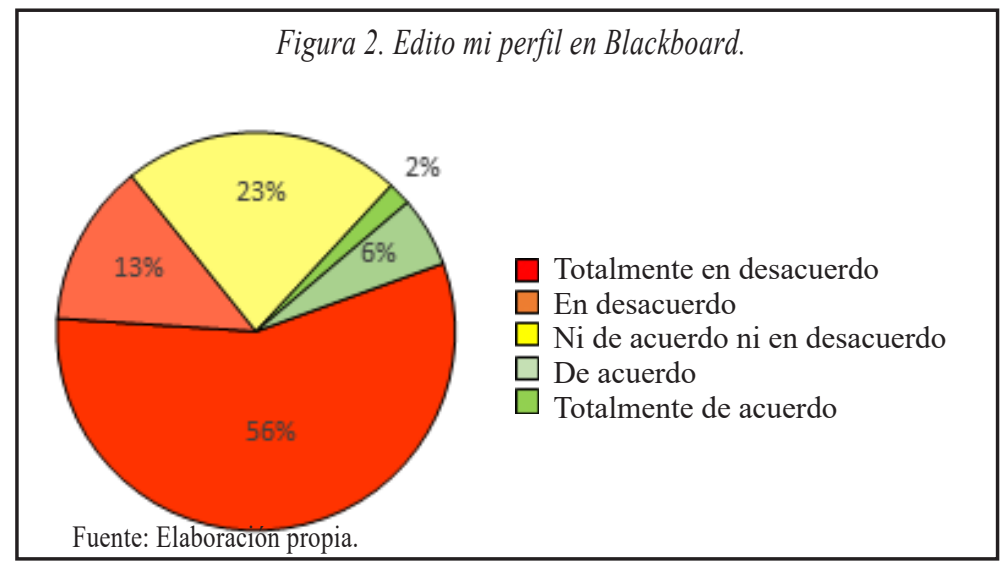


tecnología, también sabrán fortalecer sus conocimientos con las plataformas educativas.

2. DI-DE (0.527). Las dimensiones instrumental y emocional tienen un grado de correlación moderado, que podría indicar que el dominio técnico de una tecnología permite favorecer el equilibrio afectivo-personal al usar la plataforma. De hecho, esto también puede mostrar que en la medida que un alumno tenga mayor dominio en el uso de la tecnología, se sentirá más confortable utilizándola como parte de su aprendizaje.

3. DI-HT (0.321). La dimensión instrumental y las herramientas tecnológicas tienen un grado de correlación relativamente bajo. Este resultado podría deberse a que los estudiantes están dispuestos a aprender usando tecnología, pero no necesariamente con Blackboard, por lo que sería muy valioso explorar qué ven de útil y amigable en otras herramientas tecnológicas.

4. DI-AL (0.260). La dimensión instrumental y aprendiendo en línea tienen un grado de correlación bajo. Esto podría indicar que los estudiantes conocen la existencia de las herramientas de Blackboard; sin embargo, tienen dificultades para usarlas.

\section{Dimensión coggnitivi}

La concepción del alumno como sujeto activo procesador de información del paradigma cognitivo constituye un aspecto básico en la educación a distancia. Rivas (2008, p. 71) explica que la cognición "entraña procesos de adquisición, transformación, organización, retención y uso de la información". Los estudiantes deben extraer la información del entorno digital en el que trabajan para procesar, adquirir y usar nuevos conocimientos. Para lograrlo deberán sentirse identificados y cómodos estudiando con el apoyo de tecnología. Por lo anterior, se indagó si les interesa aprender usando plataformas educativas. A pesar de que son estudiantes de la generación de nativos digitales, los resultados mostraron que solamente el $53 \%$ está de acuerdo 0 totalmente de acuerdo (ver fig. 3).

Blackboard ofrece un entorno personal para aprender que Adell y Castañeda (2010, p. 7) definen como "conjunto de herramientas, fuentes de información, conexiones y actividades que cada persona utiliza de forma asidua para aprender". Al no

Figura 3. Me interesa aprender usando plataformas educativas.

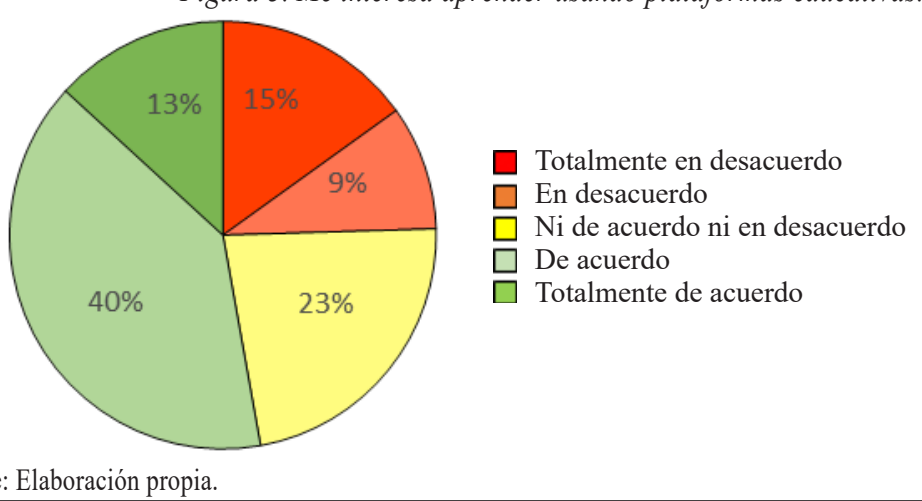


estar interesados en aprender usando plataformas educativas se dificultará el proceso cognitivo y la formación de futuros ciudadanos de un mundo digital.

Area (2012, p. 30) explica que la dimensión cognitiva implica "aprender a utilizar de forma inteligente la información para acceder a la misma, otorgarle significado, analizarla críticamente y reconstruirla personalmente" y también incluye la comunicación usando recursos digitales. Por lo anterior, se preguntó si les interesa usar herramientas tecnológicas que sirvan para acceder a información (y que conlleva el análisis y reconstrucción de esta) para elaborar trabajos académicos tales como Dropbox, Google Drive, Delicious, Blogger, Survey Monkey, Audacity, etcétera, y el porcentaje de alumnos interesados en ellas fue mayor (70\%) comparado con el dato concerniente a las plataformas educativas (ver figura 4).

El uso de diferentes herramientas tecnológicas favorece el desarrollo de habilidades para la adquisición, organización y uso del conocimiento, y la cognición permite integrar la información que se adquiere a través de los sentidos. La información se produce, se distribuye y se consume aceleradamente, por lo que "la capacidad para usar las tecnologías de la información es cada día más determinante" (Pérez, 2013, p. 53). Por lo anterior, el desarrollo de la dimensión cognitiva es importante para construir una base para anclar los conocimientos instrumentales y fomentar actitudes positivas hacia la tecnología a través de actividades que permitan adquirir las competencias útiles para la vida. Como señala Pérez (2013, p. 69), "el reto de la escuela contemporánea se sitúa en la dificultad y necesidad de transformar el torrente desordenado y fragmentario de informaciones en conocimiento".

La creación, búsqueda, almacenamiento, recuperación y transferencia de la información es una actividad central en las empresas turísticas (Elżbieta, 2016), por lo que la aceptación y uso de herramientas tecnológicas educativas que fomenten el manejo inteligente de la información es necesario en los programas de las carreras de turismo.

Ante la pregunta: ¿aprendo mejor en línea que de manera presencial?, solamente el $6 \%$ estuvo de acuerdo (ver figura 5). Este dato refleja que los estudiantes no han aceptado ni adoptado a Blackboard como un medio para su aprendizaje. Tal situación podría explicarse gracias a la teoría de aceptación y uso de la tecnología (UTAUT), cuyo modelo incluye cuatro constructos: 1) expectativa de rendimiento: percepción sobre el grado de utilidad de la tecnología; 2) expectativa de esfuerzo: la facilidad

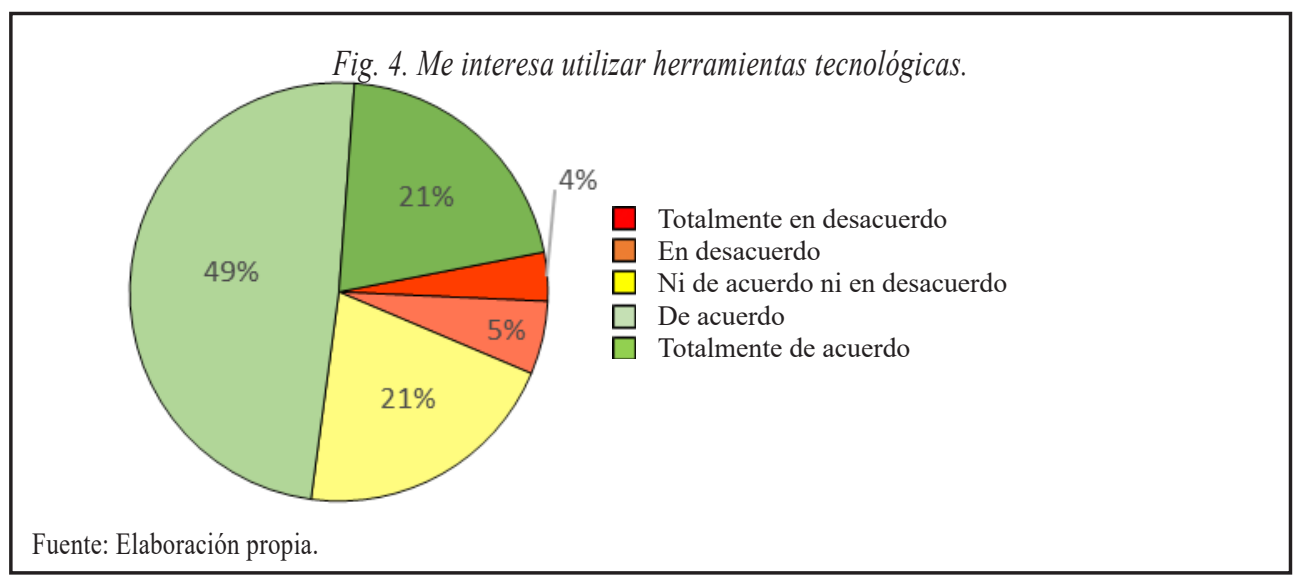




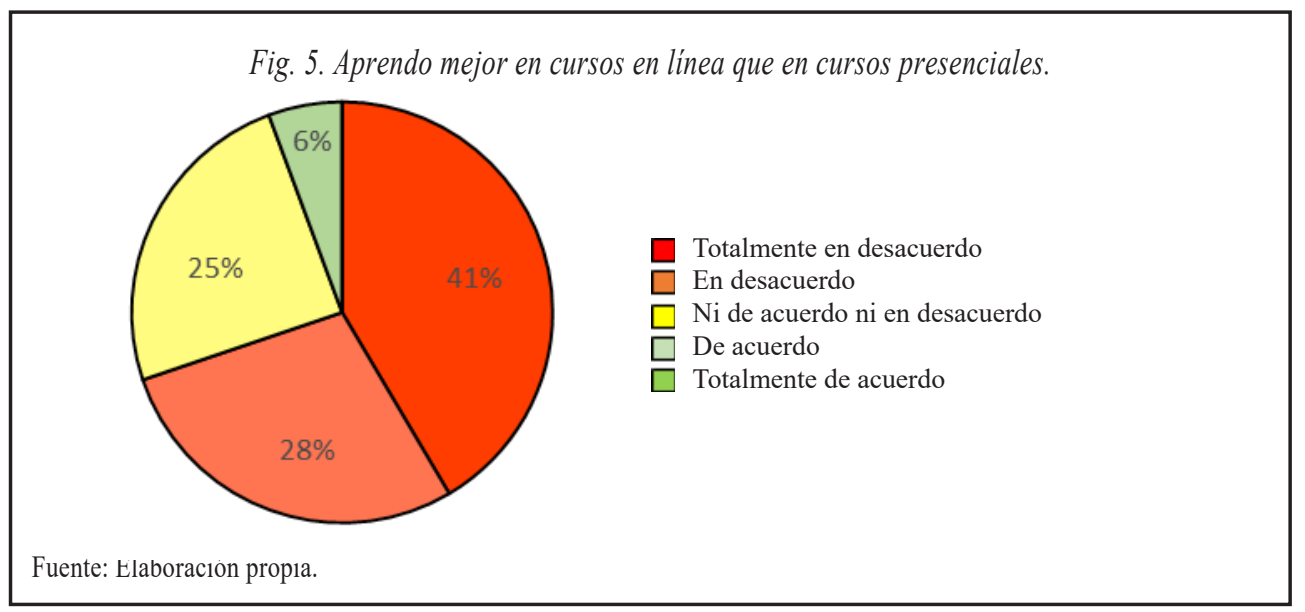

de uso de las tecnologías; 3) factores sociales: la medida en que los individuos piensan que otras personas importantes creen que deberían usar las tecnologías; $y$, 4) condiciones de facilitación: el grado percibido en que existe la infraestructura organizacional y técnica requerida para el soporte de las tecnologías (Thomas, Singh y Gaffar, 2013).

Del análisis correlacional de la dimensión cognitiva se obtuvieron los resultados mostrados en la tabla 2.

Interpretación de las correlaciones de la dimensión cognitiva:

1. DC-DI (0.518). Se observó que ambas dimensiones tienen un grado de correlación moderado, que podría indicar que el dominio técnico de una tecnología (en este caso Blackboard) facilitará el uso inteligente de la información. Dicha correlación es positiva, lo que implica también que ambas dimensiones se fortalecen mutuamente. A medida que los alumnos se consideran buenos con el uso de la tecnología también sabrán fortalecer sus conocimientos con las plataformas educativas.

2. DC-DE (0.573). Las dimensiones cognitiva y emocional mostraron una relación positiva moderada. Esto podría indicar que, al poseer conocimientos y habilidades para usar de forma inteligente la información, el equilibrio afectivo-personal en el uso de las TIC es más factible.

3. DC-HT (0.195). La correlación es positiva, pero no estadísticamente significativa, lo que implica que la relación presentada entre las dos variables no tiene relevancia desde el punto de vista estadístico.

Tabla 2. Resultados de las correlaciones de la dimensión cognitiva

\begin{tabular}{cccccc}
\hline & & $\begin{array}{c}\text { Dimensión } \\
\text { instrumental }\end{array}$ & $\begin{array}{c}\text { Dimensión } \\
\text { emocional }\end{array}$ & $\begin{array}{c}\text { Herramientas } \\
\text { tecnológicas }\end{array}$ & $\begin{array}{c}\text { Aprendiendo } \\
\text { en línea }\end{array}$ \\
\hline \multirow{2}{*}{$\begin{array}{c}\text { Dimensión } \\
\text { cognitiva }\end{array}$} & $\begin{array}{c}\text { Correlación } \\
\text { de Pearson }\end{array}$ & $0.518^{* *}$ & $0.573^{* *}$ & 0.195 & -0.022 \\
\cline { 2 - 6 } & $\begin{array}{c}\text { Sig. } \\
\text { (bilateral) }\end{array}$ & 0.000 & 0.000 & 0.161 & 0.875 \\
\cline { 2 - 6 } & $\mathrm{N}$ & 53 & 53 & 53 & 53 \\
\hline
\end{tabular}

24 *Significativa al 0.05 bilateral. **Significativa al 0.01 bilateral.

Fuente: Elaboración propia. 
4. DC-AL (-0.022). Un resultado destacable de esta correlación es su signo negativo, que refuerza lo que se ha presentado en otras correlaciones: a pesar de que los alumnos tienen a su disposición herramientas para el aprendizaje, se les dificulta el uso de la herramienta Blackboard. La correlación no es significativa; es decir, entre estas dos variables no hay ninguna relación.

\section{Dimensión emocionial}

Como lo señala Mora (2013), las emociones son muy importantes para sustentar el aprendizaje, y por ello se preguntó a los estudiantes si se sienten contentos estudiando en línea. Solamente el $28 \%$ declaró que se siente contento y $42 \%$ no pudo definir si se siente contento o no (ver figura 6). Tales resultados requieren especial atención, ya que la experiencia emocional es un componente que influye en los procesos de alfabetización digital. Conocer cómo se sienten estudiando en línea es una información valiosa debido a que "la simple exposición a las TIC no genera estados emocionales relevantes en los usuarios" (Ricoy y Fernández, 2013, p. 512). Diversas variables intervienen en las respuestas emocionales, y el hecho de que una minoría se sienta contenta aprendiendo en línea podría revelar varias situaciones, entre ellas que no han sido formados para una modalidad de este tipo, prefieren el contacto presencial con el docente o no saben utilizar la tecnología en beneficio de su aprendizaje. Este hallazgo es significativo debido a que una emoción negativa favorece el rechazo a la tecnología como mediadora del aprendizaje y es portadora de un significado que permitirá implementar acciones para una apropiación tecnológica emocionalmente positiva.

La exploración de las emociones de los estudiantes en línea constituye un elemento nuclear para analizar los procesos de alfabetización digital en las universidades debido a los tres principios del aprendizaje que sirven de base para argumentar el papel que juega la inteligencia emocional como predictor positivo del éxito académico. Tales principios refieren que todo aprendizaje tiene una base emocional, que las emociones que aportan mayores beneficios son las positivas y que el aprendizaje humano es fundamentalmente social (Bisquerra, Pérez y García, 2015).

Los estudiosos de las emociones han planteado diferentes modelos para analizarlas, y diversos autores, entre los que se encuentran Rusell, Feldman, Barrett, Larsen

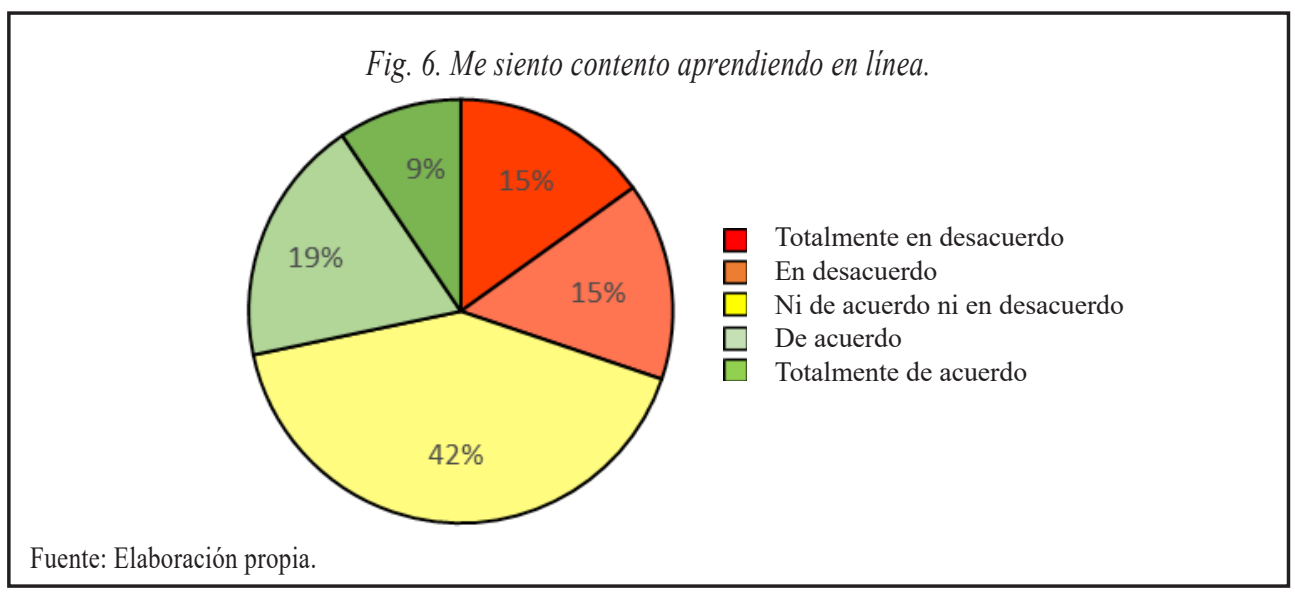


Fig. 7. Modelo circumplejo.

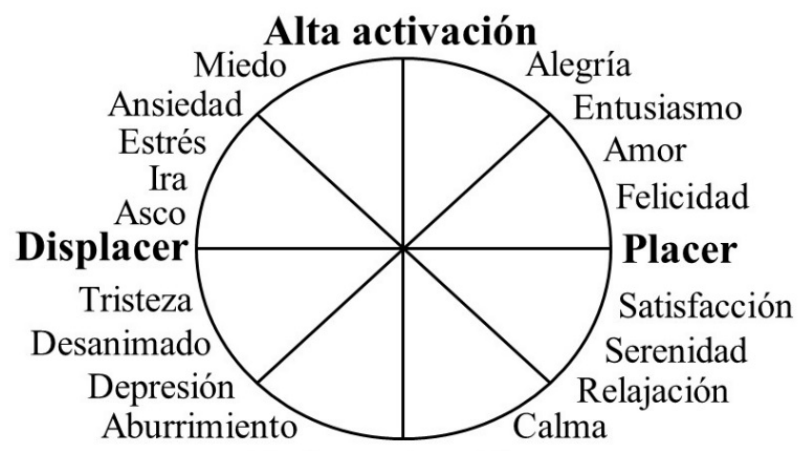

Baja activación

Fuente: Bisquerra, J.C Pérez y E. García, 2015, p. 145.

y Diener, propusieron un modelo circumplejo (ver figura 7) con las dimensiones de activación o desactivación y las dimensiones de lo desagradable o negativo y lo agradable o positivo (Bisquerra, Pérez y García, 2015).

Dado que una de las preguntas del cuestionario aborda una emoción positiva, se determinó preguntar también acerca de una emoción ubicada en la dimensión de lo desagradable. Los resultados sobre esa emoción mostraron que el 39\% de los estudiantes piensa que estudiar en línea es aburrido (ver fig. 8).

Pekrun, Goetz y Titz (2010), en su investigación sobre emociones académicas, refieren que el aburrimiento funciona como escape conductual o mental de situaciones que no aportan un estímulo suficiente, o bien sirve de escape de situaciones que sobrepasan las habilidades de los estudiantes. Considerando lo expresado por estos autores, se indagó cómo se sienten los estudiantes acerca de sus habilidades para aprender en línea y sus respuestas mostraron que el 79\% está consciente de que tienen que mejorarlas (ver figura 9). Al contrastar este dato con el porcentaje de estudiantes que piensan que estudiar en línea es aburrido, cabe la posibilidad de que el sentimiento que experimentan tiene como origen habilidades insuficientes para esta modalidad.

El resultado obtenido en esta pregunta coincide con otras investigaciones sobre la incorrecta suposición de que los estudiantes están familiarizados con las TIC para

Fig. 8. Es aburrido estudiar en linea.

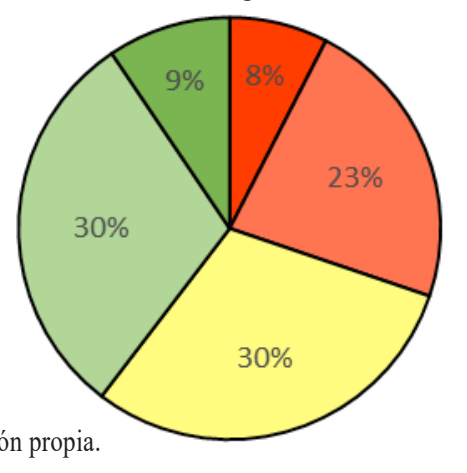

Totalmente en desacuerdo

En desacuerdo

Ni de acuerdo ni en desacuerdo

De acuerdo

Totalmente de acuerdo

Fuente: Elaboración propia. 
su uso en contextos formales (Berrío Zapata y Rojas, 2014; Bossolasco y Storni, 2012; Godoy-Rodríguez, 2009; ICDL Américas, 2015; IEA, 2014).

Del análisis correlacional de la dimensión emocional se obtuvieron los resultados mostrados en la tabla 3.

\begin{tabular}{|c|c|c|c|c|c|}
\hline & & $\begin{array}{c}\text { Dimensión } \\
\text { instrumental }\end{array}$ & $\begin{array}{c}\text { Dimensión } \\
\text { cognitiva }\end{array}$ & $\begin{array}{c}\text { Herramientas } \\
\text { tecnológicas }\end{array}$ & $\begin{array}{c}\text { Aprendiendo } \\
\text { en línea }\end{array}$ \\
\hline \multirow{3}{*}{$\begin{array}{l}\text { Dimensión } \\
\text { emocional }\end{array}$} & $\begin{array}{c}\text { Correlación de } \\
\text { Pearson }\end{array}$ & $0.527 * *$ & $0.573 * *$ & $0.325^{*}$ & 0.035 \\
\hline & Sig. (bilateral) & 0.000 & 0.000 & 0.018 & 0.803 \\
\hline & $\mathrm{N}$ & 53 & 53 & 53 & 53 \\
\hline
\end{tabular}

*Significativa al 0.05 bilateral. **Significativa al 0.01 bilateral.

Fuente: Elaboración propia.

Interpretación de las correlaciones de la dimensión emocional:

1. DE-DI (0.527). Las dimensiones instrumental y emocional tienen un grado de correlación moderado, que podría indicar que el dominio técnico de una tecnología permite favorecer el equilibrio afectivo-personal al usar la plataforma. De hecho, esto también puede mostrar que en la medida en que un alumno tenga mayor dominio en el uso de la tecnología se sentirá más confortable utilizándola como parte de su aprendizaje.

2. DE-DC (0.573). Las dimensiones cognitiva y emocional mostraron una relación positiva moderada. Esto podría indicar que, al poseer conocimientos y habilidades para usar de forma inteligente la información, el equilibrio afectivo-personal en el uso de las TIC es más factible.

3. DE-HT (0.325). La correlación entre la dimensión emocional y las habilidades tecnológicas es moderadamente significativa, lo cual permite inferir que si los alumnos se sienten a gusto con la tecnología estarán más dispuestos a aprender con ella; 0 viceversa, estar en contacto continuo con la tecnología les facilitará sentirse seguros utilizándola para el aprendizaje.

4. DE-AL (0.035). La relación entre la dimensión emocional y el aprendizaje en línea es muy pequeña (cercana a cero) y además no es significativa. Cabe destacar

Figura 9. Estoy consciente de que tengo que mejorar mis habilidades para aprender en línea.

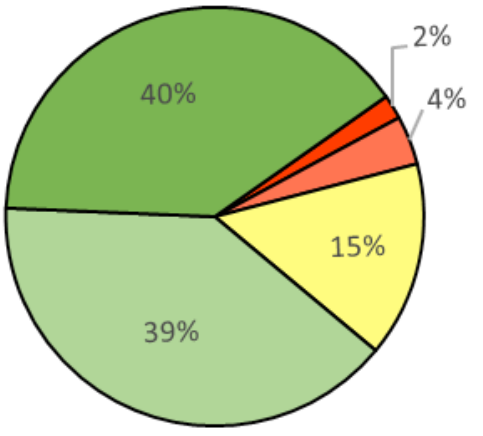

Totalmente en desacuerdo

En desacuerdo

Ni de acuerdo ni en desacuerdo

De acuerdo

Totalmente de acuerdo

Fuente: Elaboración propia. 
que al parecer el aprendizaje en la plataforma evaluada no genera bienestar en el grupo de alumnos evaluados.

\section{Iler'rimientias teconológicials}

A través del cuestionario también se exploraron las preferencias de los estudiantes sobre algunas de las herramientas tecnológicas más usadas para propósitos educativos que se encuentran disponibles en la red. Los resultados se muestran en la tabla 4.

\begin{tabular}{llc}
\hline \multicolumn{3}{l}{ Tabla 4. Preferencias de herramientas } \\
\hline No. & Herramienta tecnológica & $\mathbf{\%}$ \\
\hline 1 & Video & $77 \%$ \\
\hline 2 & Tutoriales & $70 \%$ \\
\hline 3 & Páginas web & $64 \%$ \\
\hline 4 & Libro electrónico & $38 \%$ \\
\hline 5 & Podcast & $30 \%$ \\
\hline Fuente: & Elaboración propia.
\end{tabular}

La herramienta preferida por los estudiantes refleja la inclinación que tienen para usar el sentido de la vista y el auditivo para aprender conceptos y procedimientos. En el caso de los tutoriales, esta misma tendencia se hace evidente, ya que en ellos se pueden combinar elementos visuales, auditivos e interactividad, lo que permite que sean más atractivos. Un porcentaje muy bajo de estudiantes eligió el libro electrónico, lo que confirma que en el aprendizaje en línea es necesario incorporar, además de los libros, recursos multisensoriales para activar más de un sentido, porque "hay formas diversas de percibir, de apreciar, de mirar y de sentir" (Barros y Barros, 2015, p. 27).

En relación con el interés por usar el podcast para el aprendizaje, solamente el $30 \%$ lo eligió como herramienta con la que le gusta aprender, esto a pesar de que los podcasts pueden ser usados para ayudar a reducir los sentimientos de soledad y contribuir a la presencial social (Lee y Chan, 2007).

Del análisis correlacional de las herramientas tecnológicas se obtuvieron los resultados mostrados en la tabla 5 .

Interpretación de las correlaciones de las herramientas tecnológicas:

1. HT-DI (0.321). La dimensión instrumental y las herramientas tecnológicas tienen un grado de correlación relativamente bajo. Este resultado podría deberse a que los estudiantes están dispuestos a aprender usando tecnología, pero no necesa-

Tabla 5. Resultados de las correlaciones de las herramientas tecnológicas

\begin{tabular}{lccccc}
\hline & & $\begin{array}{c}\text { Dimensión } \\
\text { instrumental }\end{array}$ & $\begin{array}{c}\text { Dimensión } \\
\text { cognitiva }\end{array}$ & $\begin{array}{c}\text { Dimensión } \\
\text { emocional }\end{array}$ & $\begin{array}{c}\text { Aprendiendo } \\
\text { en línea }\end{array}$ \\
\hline \multirow{2}{*}{$\begin{array}{l}\text { Herramientas } \\
\text { tecnológicas }\end{array}$} & $\begin{array}{c}\text { Correlación de } \\
\text { Pearson }\end{array}$ & $0.321^{*}$ & 0.195 & $0.325^{*}$ & -0.041 \\
\cline { 2 - 6 } & Sig. (bilateral) & 0.019 & 0.161 & 0.018 & 0.769 \\
\cline { 2 - 6 } & $\mathrm{N}$ & 53 & 53 & 53 & 53 \\
\hline
\end{tabular}

28 *Significativa al 0.05 bilateral. **Significativa al 0.01 bilateral.

Fuente: Elaboración propia. 
riamente con Blackboard, por lo que sería muy valioso explorar qué ven de útil y amigable en otras herramientas tecnológicas.

2. HT-DC (0.195). La correlación es positiva, pero no estadísticamente significativa, lo que implica que la relación presentada entre las dos variables no tiene relevancia desde el punto de vista estadístico.

3. HT-DE (0.325). La correlación entre la dimensión emocional y las habilidades tecnológicas es moderadamente significativa, lo cual permite inferir que si los alumnos se sienten a gusto con la tecnología estarán más dispuestos a aprender con ella; o viceversa, estar en contacto continuo con la tecnología les facilitará sentirse seguros utilizándola para el aprendizaje.

4. HT-AL (-0.041). Esta correlación muestra gran parte de los resultados anteriores. Los alumnos tienen habilidades tecnológicas, pero no se ven reflejadas en el aprendizaje en línea usando Blackboard. Lo anterior tiene implicaciones relevantes como: 1) a pesar de que los alumnos están en contacto con la tecnología, no se han apropiado de ella para procesos de aprendizaje; y, 2) Blackboard les resulta una herramienta complicada de utilizar.

\section{Aprendiendo en línęi}

En un EVEA se manejan diferentes herramientas tecnológicas que los estudiantes deben usar en beneficio de su aprendizaje. De igual manera, aprender en línea también implica administrar el tiempo para estudiar contenidos y realizar actividades. Por lo anterior, se consideró importante conocer si tienen dificultades para el estudio con el apoyo de Blackboard (ver tabla 6).

\begin{tabular}{llc}
\hline \multicolumn{3}{c}{ Tabla 6. Dificultades para el estudio en EVEA } \\
\hline No. & Dificultad & $\%$ \\
\hline 1 & Consulta de calificaciones & $70 \%$ \\
\hline 2 & Leer comentarios en actividades & $62 \%$ \\
\hline 3 & Subir actividades & $58 \%$ \\
\hline 4 & Usar el menú global & $57 \%$ \\
\hline 5 & Participar en el tablero de discusión & $55 \%$ \\
\hline 6 & Administración del tiempo & $47 \%$ \\
\hline 5 & Usar el correo electrónico & $46 \%$ \\
\hline 7 & Participar en videoconferencias en & $40 \%$ \\
\hline
\end{tabular}

Fuente: Elaboración propia.

Los resultados hacen evidente que más del 50\% tiene dificultades de manera frecuente o muy frecuente para subir las actividades a Blackboard, consultar calificaciones y leer los comentarios de los maestros sobre sus trabajos. Como consecuencia de no estar en condiciones de cumplir con la entrega de sus trabajos, no dar seguimiento a la retroalimentación de los maestros y no estar al tanto de su desempeño académico es factible que los estudiantes experimenten situaciones de estrés que afecten la experiencia educativa en línea. 
Además de las herramientas disponibles dentro del EVEA utilizado por la universidad, se consideró pertinente explorar si los estudiantes utilizan otras herramientas para el desarrollo de sus actividades académicas (ver tabla 7). Se les preguntó sobre cuatro herramientas con diferentes funciones: para elaboración de trabajos colaborativos en línea, para almacenamiento en la nube, para comunicación síncrona y una red social con diversos fines didácticos.

Se pudo confirmar que una de las herramientas más utilizadas es el software para videoconferencias que utilizan para comunicarse con sus compañeros en mayor medida que con sus profesores. Este dato contrasta significativamente con la información obtenida sobre el uso de la herramienta para videoconferencias Blackboard Collaborate (ver tabla 6), debido a que el $40 \%$ de los estudiantes respondió que tiene problemas para usarla. Este hallazgo podría significar que los estudiantes desconocen cómo usarla o que otras herramientas son más amigables para la misma función.

Cabe señalar que $43 \%$ de los estudiantes hacen uso de herramientas de almacenamiento en la nube y $38 \%$ usa aplicaciones de Google para trabajos colaborativos, lo que significa que además del EVEA de la institución, los estudiantes conocen y usan otras herramientas para llevar a cabo sus actividades académicas.

Del análisis correlacional sobre "aprendiendo en línea" se obtuvieron los resultados que se muestran en la tabla 8 .

Interpretación de las correlaciones de aprendiendo en línea:

1. AL-DI (0.260). La dimensión instrumental y aprendiendo en línea tienen un grado de correlación bajo. Esto podría indicar que los estudiantes conocen la existencia de las herramientas de Blackboard; sin embargo, tienen dificultades para usarlas.

2. AL-DC (-0.022). Un resultado destacable de esta correlación es su signo negativo que refuerza lo que se ha presentado en otras correlaciones: a pesar de que los alumnos tienen a su disposición herramientas para el aprendizaje, se les dificulta el uso de la herramienta Blackboard. La correlación no es significativa; es decir, entre estas dos variables no hay ninguna relación.

3. AL-DE (0.035). La relación entre la dimensión emocional y el aprendizaje en línea es muy pequeña (cercana a cero) y además no es significativa. Cabe destacar que al parecer el aprendizaje en la plataforma evaluada no genera bienestar en el grupo de alumnos evaluados.

\begin{tabular}{lccccc}
\hline \multicolumn{6}{c}{ Tabla 7. Herramientas utilizadas para actividades académicas } \\
\hline \multicolumn{7}{l}{} & $\mathbf{1}$ & $\mathbf{2}$ & $\mathbf{3}$ & $\mathbf{4}$ & $\mathbf{5}$ \\
\hline $\begin{array}{l}\text { Uso Google Docs para mis trabajos } \\
\text { académicos colaborativos en línea. }\end{array}$ & $26.4 \%$ & $11.3 \%$ & $16.9 \%$ & $20.7 \%$ & $24.5 \%$ \\
\hline Acostumbro a guardar archivos en la nube. & $28.3 \%$ & $15.0 \%$ & $16.9 \%$ & $13.2 \%$ & $26.4 \%$ \\
\hline $\begin{array}{l}\text { Uso software de videoconferencias para } \\
\text { comunicarme con mis maestros. }\end{array}$ & $28.3 \%$ & $7.5 \%$ & $13.2 \%$ & $20.7 \%$ & $30.1 \%$ \\
\hline $\begin{array}{l}\text { Uso software de videoconferencias para } \\
\text { comunicarme con mis compañeros. }\end{array}$ & $41.5 \%$ & $13.2 \%$ & $11.3 \%$ & $7.5 \%$ & $26.4 \%$ \\
\hline Elaboro trabajos académicos con Pinterest. & $20.7 \%$ & $3.7 \%$ & $11.3 \%$ & $11.3 \%$ & $52.8 \%$ \\
\hline $\begin{array}{l}\text { Escala: 1=muy frecuentemente, 2=frecuentemente, } 3=\text { ocasionalmente, 4=raramente, 5=nunca. } \\
\text { Nota: Elaboración propia. }\end{array}$
\end{tabular}




\begin{tabular}{llcccc}
\hline & \multicolumn{4}{l}{ Tabla 8. Resultados de las correlaciones de aprendiendo en línea } & \\
\hline & & $\begin{array}{c}\text { Dimensión } \\
\text { instrumental }\end{array}$ & $\begin{array}{c}\text { Dimensión } \\
\text { cognitiva }\end{array}$ & $\begin{array}{c}\text { Dimensión } \\
\text { emocional }\end{array}$ & $\begin{array}{c}\text { Herramientas } \\
\text { tecnológicas }\end{array}$ \\
\hline \multirow{2}{*}{$\begin{array}{l}\text { Aprendiendo } \\
\text { en línea }\end{array}$} & $\begin{array}{l}\text { Correlación de } \\
\text { Pearson }\end{array}$ & 0.260 & -0.022 & 0.035 & -0.041 \\
\cline { 2 - 6 } & Sig. (bilateral) & 0.060 & 0.875 & 0.803 & 0.769 \\
\cline { 2 - 6 } & $\mathrm{N}$ & 53 & 53 & 53 & 53 \\
\hline
\end{tabular}

*Significativa al 0.05 bilateral. **Significativa al 0.01 bilateral.

Fuente: Elaboración propia.

4. AL-HT (-0.041). Esta correlación muestra gran parte de los resultados anteriores. Los alumnos tienen habilidades tecnológicas, pero no se ven reflejadas en el aprendizaje en línea usando Blackboard. Lo anterior tiene implicaciones relevantes como: 1) a pesar de que los alumnos están en contacto con la tecnología, no se han apropiado de ella para procesos de aprendizaje; y, 2) Blackboard les resulta una herramienta complicada de utilizar.

\section{Conculsones}

Analizar la alfabetización digital desde la perspectiva de la dimensión instrumental mostró que hay estudiantes que no saben usar el correo electrónico de Blackboard y que menos de la mitad lo utilizan para comunicarse con sus maestros. De igual forma, son pocos los estudiantes que editan su perfil, situación que afecta la construcción de una identidad digital. En cuanto a la dimensión cognitiva, a pesar de ser nativos digitales, todavía hay estudiantes que no están interesados en aprender usando plataformas educativas ni utilizar herramientas tecnológicas, y muy pocos perciben que aprenden mejor en cursos en línea que en los presenciales. En el aspecto emocional, son pocos los alumnos que se sienten contentos aprendiendo en línea y un número significativo piensa que es aburrido. La mayoría está consciente de que tienen que mejorar sus habilidades para aprender en línea.

En el análisis correlacional destacó la que existe entre las dimensiones cognitiva y emocional, lo que indica que poseer conocimientos y habilidades para usar de manera inteligente la información facilita la aceptación y adopción de las TIC para el aprendizaje.

Ser nativo digital no implica que el estudiante posea los conocimientos y las habilidades necesarias para usar las TIC en beneficio de su aprendizaje; por tal razón, las universidades deben capacitar a los alumnos para que acepten y adopten el EVEA en el que trabajarán antes de ingresar a las asignaturas en línea. Para lograrlo, es recomendable que, teniendo como guía la teoría de aceptación y uso de la tecnología, se cercioren que los jóvenes universitarios conozcan el grado de utilidad de la tecnología, reciban la formación necesaria para facilitarles el uso de las herramientas con las que trabajarán y que la infraestructura organizacional apoye con el soporte necesario. 
Para que los estudiantes universitarios tengan experiencias satisfactorias estudiando en línea, el conocimiento de las herramientas del EVEA es fundamental; sin embargo, también es necesario que exista comunicación interpersonal de calidad entre docentes y estudiantes, así como entre los mismos estudiantes, interactividad con el apoyo de herramientas de la web 2.0 e integración de los aprendices en forma dinámica y activa.

Para facilitar la adopción de los EVEA es necesario implementar acciones que conduzcan a fortalecer la dimensiones instrumental, cognitiva y emocional para que adquieran los conocimientos, habilidades, disposiciones y conductas necesarios para desenvolverse con éxito en el mundo digital educativo.

Las TIC tienen un valor estratégico en la formación de profesionales que trabajarán en la industria del turismo debido a que la tecnología se ha extendido por las ventajas que ofrece para la gestión administrativa, distribución de productos turísticos, optimización de procesos y aplicación de mercadotecnia digital. En consecuencia, formarlos en el uso de las TIC educativas contribuirá a que aprendan a aceptar y adoptar tecnologías para usos críticos y productivos.

\section{REFRREIIIS}

Adell, J. y Castañeda, L. (2010). Los entornos personales de aprendizaje (PLES): una nueva manera de entender el aprendizaje. Recuperado de https://digitum.um.es/jspui/ bitstream/10201/17247/1/Adell\&Casta\%C3\%B1eda 2010.pdf

Area, M. (2012). La alfabetización en la sociedad digital. En Alfabetización digital y competencias informacionales. Madrid, España: Fundación Telefónica/Editorial Ariel. Recuperado de https://ddv.stic.ull.es/users/manarea/public/libro_\%20Alfabetizacion_digital.pdf

Area, M. (2015). La alfabetización digital y la formación de la ciudadanía del siglo XXI. Recuperado de http://www.scielo.org.bo/pdf/rieiii/v7n3/v7n3 a02.pdf

ANUIES. (2001). Diagnóstico de la educación superior a distancia en México. México: ANUIES. ANUIES. (2015). Diagnóstico de la educación superior a distancia en México. México: ANUIES.

Barker, J. y Gossman, P. (2013). The learning impact of a virtual learning environment: students' views. Team Journal, 5(2). Recuperado de http://194.81.189.19/ojs/index.php/TEAN/article/ viewFile/146/261

Barros, C. y Barros, R. (2015, diciembre). Los medios audiovisuales y su influencia en la educación desde alternativas de análisis. Revista Universidad y Sociedad, 7(3). Recuperado de http://scielo.sld.cu/pdf/rus/v7n3/rus04315.pdf

Bawden, D. (2008). Origins and concepts of digital literacy. En C. Lankshear y M. Knobel, Digital literacies: Concepts, policies and practices. Nueva York: Peter Lang Publishing. Recuperado de http://citeseerx.ist.psu.edu/viewdoc/download?doi=10.1.1.741.4617\&rep=rep1\&type=pdf

Benavides, F. y Pedró, F. (2007). Políticas educativas sobre nuevas tecnologías en los países iberoamericanos. Revista Iberoamericana de Educación, 45. Recuperado de https://rieoei.org/ historico/documentos/rie45a01.htm

Bernal, C. (2016). Metodología de la investigación (4a ed.). Bogotá, Colombia: Pearson Educación.

Berrío Zapata, C. y Rojas, H. (2014). La brecha digital universitaria: la apropiación de las TIC en estudiantes de educación superior en Bogotá (Colombia). Revista Comunicar, XXII(43). Recuperado de https://www.revistacomunicar.com/index. php? contenido $=$ detalles\&numer $=43 \&$ articulo $=43-2014-13$

Bisquerra, R., Pérez, J.C. y García, E. (2015). Inteligencia emocional en educación. Madrid, 32 España: Editorial Síntesis. 
Bossolasco, M. y Storni, P. (2012). ¿Nativos digitales?: hacia una reflexión crítica de la construcción de los jóvenes como usuarios expertos de las nuevas tecnologías. Análisis de una experiencia de inclusión de las TIC en la escuela. RED, Revista de Educación a Distancia, 30. Recuperado de http://www.um.es/ead/red/30/bossolasco.pdf

Boston Consulting Group. (2012). The value of our digital identity. Recuperado de https://2zn23x1nwzzj494slw48aylw-wpengine.netdna-ssl.com/wp-content/uploads/2017/06/ The-Value-of-Our-Digital-Identity.pdf

Cabero, J. y Barroso, J. (coords.). (2015). Nuevos retos en tecnología educativa. Madrid, España: Editorial Síntesis.

Clarenc, C.A., Castro, S.M., López de Lenz, M.E., Moreno, M.E. y Tosco, N.B. (2013). Analizamos 19 plataformas de eLearning: investigación colaborativa sobre LMS. Congreso Virtual Mundial de e-Learning. Grupo GEIPITE. Recuperado de http://cooperacionib. org/191191138-Analizamos-19-plataformas-de-eLearning-primera-investigacion-academicacolaborativa-mundial.pdfC

CRUE-TIC y REBIUN. (2009). Competencias informáticas e informacionales en los estudios de grado. Recuperado de https://www.uv.es/websbd/formacio/ci2.pdf

Elżbieta, D. (2016). Advantages from ICT usage in hotel industry. Czech Journal of Social Sciences, Business and Economics, 5(3). Recuperado de https://www.researchgate.net/ publication/316446822_Advantages_from_ICTS_usage_in_hotel_industry

Gilster, P. (1997). Digital literacy. Estados Unidos: John Wiley \& Sons.

Godoy-Rodríguez, C. (2009). Alfabetización digital, comportamientos y percepciones respecto a las TIC de los estudiantes universitarios venezolanos. Un caso desde el estado Barinas. Revista Latinoamericana de Tecnología Educativa-RELATEC, 8(1), 83-104. Recuperado de http://relatec.unex.es/article/view/455

Hiraldo, R. (2013). Uso de los entornos virtuales de aprendizaje en la educación a distancia. Edutec. Recuperado de https://www.uned.ac.cr/academica/edutec/memoria/ponencias/ hiraldo_162.pdf

International Computer Driver License Américas (ICDL). (2015). Encuesta de competencias digitales. León, México. Recuperado de http://icdlamericas.org/media/informe_encuesta_de_ competencias_digitales_-_leon,_mexico_1.pdf

IEA (2014). Press release. Recuperado de https://www.iea.nl/sites/default/files/studies/IEA\%20 ICILS\%202013\%20Press\%20Release.pdf

IIPE-UNESCO. (2006). La integración de las tecnologías de la información y la comunicación en los sistemas educativos. Recuperado de http://unesdoc.unesco.org/ images/0015/001507/150785s.pdf

Jimoyiannis, A. y Gravani, M. (2011, enero). Exploring adult digital literacy using learners' and educators' perceptions and experiences: The case of the second chance schools in Greece. Educational Technology \& Society, 14(1). Recuperado de https://www.researchgate. net/publication/220374667_Exploring_Adult_Digital_Literacy_Using_Learners'_and Educators'Perceptions_and_Experiences_The_Case_of the_Second_Chance_Schools_in Greece

JISC. (2014). Developing digital literacies. Recuperado de https://www.jisc.ac.uk/guides/ developing-digital-literacies

Koutropoulos, A. (2011, diciembre). Digital natives: Ten years after. MERLOT Journal of Online Learning and Teaching, 7(4). Recuperado de http://jolt.merlot.org/vol7no4/ koutropoulos_1211.htm

Lee, M.J.W. y Chan, A. (2007, enero). Reducing the effects of isolation and promoting inclusivity for distance learners through podcasting. Turkish Online Journal of Distance Education, 8(1). Recuperado de http://www.acarindex.com/dosyalar/makale/acarindex-1423932372.pdf

Lynch. M. (2017). Digital literacy is the most important lifelong learning tool. Recuperado de https://www.thetechedvocate.org/digital-literacy-important-lifelong-learning-tool/

Moore, S. y Kuol, N. (2007, noviembre). Matters of the heart: Exploring the emotional dimensions of educational experience in recollected accounts of excellent teaching. International 
Journal for Academic Development, 12(2). Recuperado de https://www.researchgate.net/ publication/248943211_Matters_of the_Heart_Exploring_the_emotional_dimensions_of_ educational_experience_in_recollected_accounts_of_excellent_teaching

Mora, F. (2013). Neuroeducāión: solo se püede aprēndēr aquello que se ama. Madrid, España: Alianza Editorial.

Mortiboys, A. (2016). Cómo enseñar con inteligencia emocional: guía paso a paso para profesionales de educación media superior, superior y posgrado. México: Grupo Editorial Patria.

OCDE. (2002). Los desafios de las tecnologías de la información y las comunicaciones en la educación. Recuperado de http://www.keepeek.com/Digital-Asset-Management/oecd/ education/los-desafios-de-las-tecnologias-de-la-informacion-y-las-comunicaciones-en-laeducacion_9789264103429-es\#page1

OCDE. (2010). Habilidades y competencias del siglo XXI para los aprendices del nuevo milenio en los paises de la OCDE. Recuperado de http://recursostic.educacion.es/blogs/europa/media/ blogs/europa/informes/Habilidades_y_competencias_siglo21_OCDE.pdf

OCDE. (2015). Habilidades para el progreso social: el poder de las habilidades sociales y emocionales. Recuperado de http://www.oecd.org/education/ceri/skills-for-social-progressexecutive-summary-spanish.pdf

Pekrun, R., Goetz, T. y Titz, W. (2010, enero). Academic emotions in students' self-regulated learning and achievement: A program of qualitative and quantitative research. Educational Psychologist, 37 (2). Recuperado de https://www.tandfonline.com/doi/abs/10.1207/ S15326985EP3702 4

Pérez, M. (2009). Uso e éducativo del correo electrónico. Universidad Nacional Experimental Francisco de Miranda. Recuperado de https://es.scribd.com/doc/72475833/Uso-Educativodel-Correo-Electronico

Pérez, A. (2013). La era digital: nuevos desafíos educativos. Sinéctica, 40. Recuperado de https:// sinectica.iteso.mx/index.php/SINECTICA/article/view/51/43

Poblete, M. y Villa, A. (2013). Aprendizaje basado en competencias: una propuesta para la evaluación de las competencias genéricas ( $3^{\mathrm{a}}$ ed.). Bilbao, España: Ediciones Mensajero.

Prieto, A., Lloris, A. y Torres, J.C. (2002). Introducción a la informática (3a ed.). España: McGraw Hill Interamericana de España.

Puerta Gil, C. y Sánchez Upegui, A. (2010). El correo electrónico: herramienta que favorece la interacción en ambientes educativos virtuales. Revista Virtual Universidad Católica del Norte, (30). Recuperado de https://www.redalyc.org/pdf/1942/194214476003.pdf

Ricoy, M.C. y Fernández, J. (2013). Contribuciones y controversias que genera el uso de las TIC en la educación superior: un estudio de caso. Revista de Educación, (360). Recuperado de https://www.mecd.gob.es/dctm/revista-de-educacion/articulosre360/re36023. pdf?documentId=0901e 72b814a77f7

Rivas, M. (2008). Procesos cognitivos y aprendizaje significativo. Madrid, España: Subdirección General de Inspección Educativa de la Viceconsejería de Organización Educativa de la Comunidad de Madrid. Recuperado de http://www.deposoft.com.ar/repo/publicaciones/ A9R6652.pdf

Rodríguez, T. (2008). El valor de las emociones para el análisis cultural. Papers, (87). Recuperado de https://ddd.uab.cat/pub/papers/02102862n87/02102862n87p145.pdf

Salman, T. (2015). Who am I online? Cultivating students' digital identity practices. Recuperado de http://repository.cityu.edu/bitstream/handle/20.500.11803/571/Chap4Advances. pdf? sequence $=2 \&$ is Allowed $=y$

Sanz de Acedo, M.L. (2010). Competencias cognitivas en educación superior. Madrid, España: Narcea Ediciones.

Thüring, M. y Mahlke, S. (2007, agosto). Usability, aesthetics and emotions in human-technology interaction. International Journal of Psychology, 42(4). Recuperado de https://www. researchgate.net/publication/247509276_Usability_aesthetics_and_emotions_in_humantechnology_interaction 
Thomas, T.D., Singh, L y Gaffar, K. (2013). The utility of the UTAUT model in explaining mobile learning adoption in higher education in Guyana. International Journal of Education and Development Using Information and Communication Technology (IJEDICT), 9(3). Recuperado de https://files.eric.ed.gov/fulltext/EJ1071379.pdf

UNESCO. (2013). Enfoques estratégicos sobre las TICs en educación en América Latina y el Caribe. Recuperado de http://www.unesco.org/new/fileadmin/MULTIMEDIA/FIELD/ Santiago/images/ticsesp.pdf

van den Beemt, A. (2010). Interactive media practices of young people: origins, backgrounds, motives and patterns. Oisterwijk: Uitgeverij BOXPress. Recuperado de https://pure.tue.nl/ws/ portalfiles/portal $/ 29934205$

Wosnitza, M. y Volet, S. (2005). Origin, direction and impact of emotions in social online learning. Learning and Instruction, (15), 449-464. 
